\title{
Estimation of Lost Voltage Measurements in Distribution Networks
}

\author{
Andrés Argüello, Student Member, IEEE and Gustavo Valverde, Member, IEEE \\ Electrical Power Engineering Research Laboratory \\ School of Electrical Engineering, University of Costa Rica \\ 11501-2060 UCR, San José, Costa Rica \\ andres.arguello.guillen@gmail.com, gvalverde@eie.ucr.ac.cr
}

\begin{abstract}
This paper presents an algorithm to estimate lost bus voltage measurements in balanced distribution networks regulated by centralized control schemes. The objective is to feed controllers with reliable estimations of lost measurements, using the information of similar bus voltage evolutions captured by the remaining measurements. The algorithm was tested in a 32-bus distribution network under different operating conditions.

Index Terms - Voltage control, distributed power generation, sensitivity, clustering analysis, state estimation.
\end{abstract}

\section{INTRODUCTION}

Different control schemes have been proposed in recent years to deal with voltage problems caused by the increasing penetration of Distributed Generators (DG). These schemes are classified as distributed or centralized. The distributed ones take control actions based on local information, while the centralized schemes take decisions based on measurements collected from all over the network, see for example [1], [2].

Centralized Volt/VAR control schemes may consider the use of transformer tap position, power output of DGs and capacitor banks to maintain bus voltages within desired limits. Such control schemes must be robust to model inaccuracies, measurement errors, and measurement loss. The first two are generally overcome or compensated by the controllers, but measurement loss is still an issue to deal with in centralized control schemes.

Among the contributions related to voltage control with intermittent measurements, the non-linear model predictive controller proposed in [2] is able to operate under dropped packets (lost measurements) situations. The controller is fed by estimated variables from the extended Kalman filter. The authors demonstrated the controller performance at different packet dropping rates for a 10-bus distribution network (DN), but the computation time in a larger system is uncertain.

In this paper, a simple yet effective method is proposed to approximate lost voltage measurements in centralized control schemes. To approximate any voltage measurement, the algorithm identifies clusters of bus voltages that behave similarly, and estimates the lost value from other voltage measurements in the same cluster. This algorithm does not require historical data or a state estimator to approximate lost measurements, it uses the remaining voltage measurements along with the last full set of measurements that arrived before the packet drop.

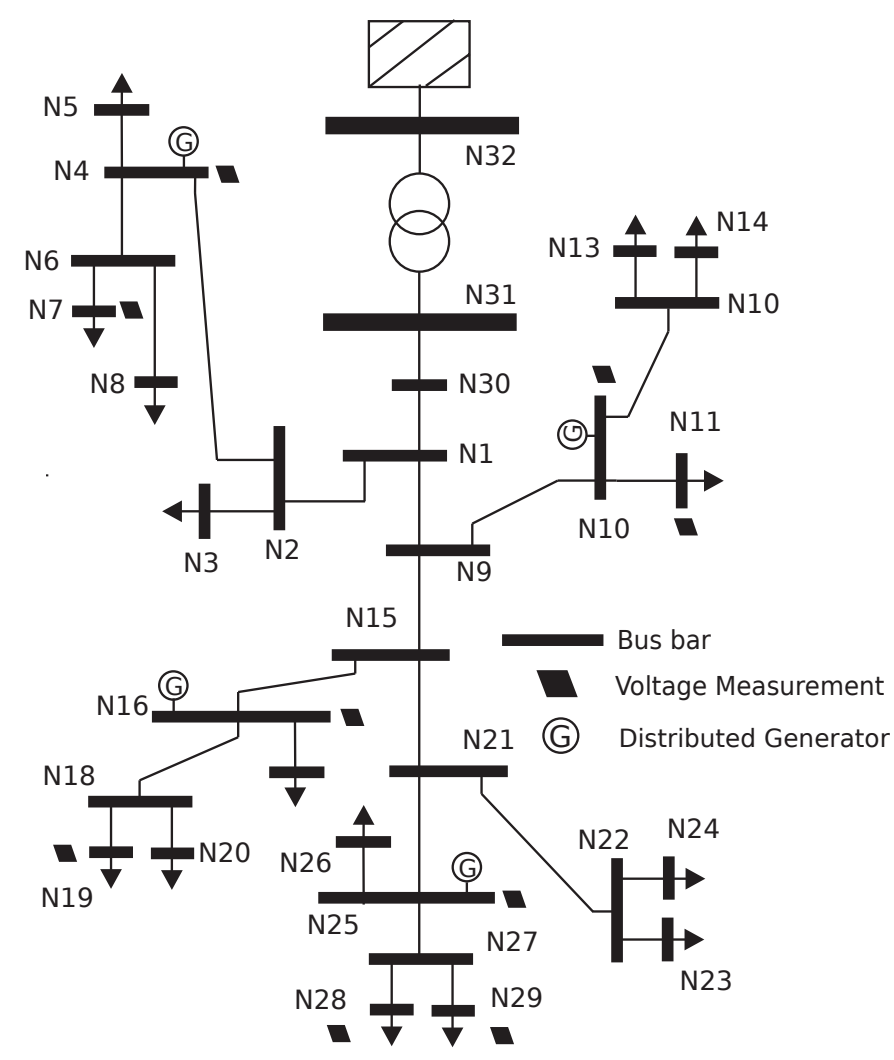

Figure 1. 32-bus test system [3]

The remaining of this paper is organized as follows: Section II introduces the principle of the estimation method. The dissimilarity calculation and the bus clustering technique are presented in Sections III and IV, while the proposed estimation algorithm is explained in Section V. Results and conclusions are finally presented in Sections VI and VII, respectively.

\section{Algorithm Principle}

The proposed algorithm comes from the observation that identifiable bus voltages, under the action of a centralized controller, behave similarly.

Consider the 32-bus test system with four monitored DGs, presented in Fig. 1. The bus voltages are regulated by a centralized controller based on Model Predictive Control (MPC) to keep the voltages within $1.000 \leq V \leq 1.025 \mathrm{pu}$. Every 


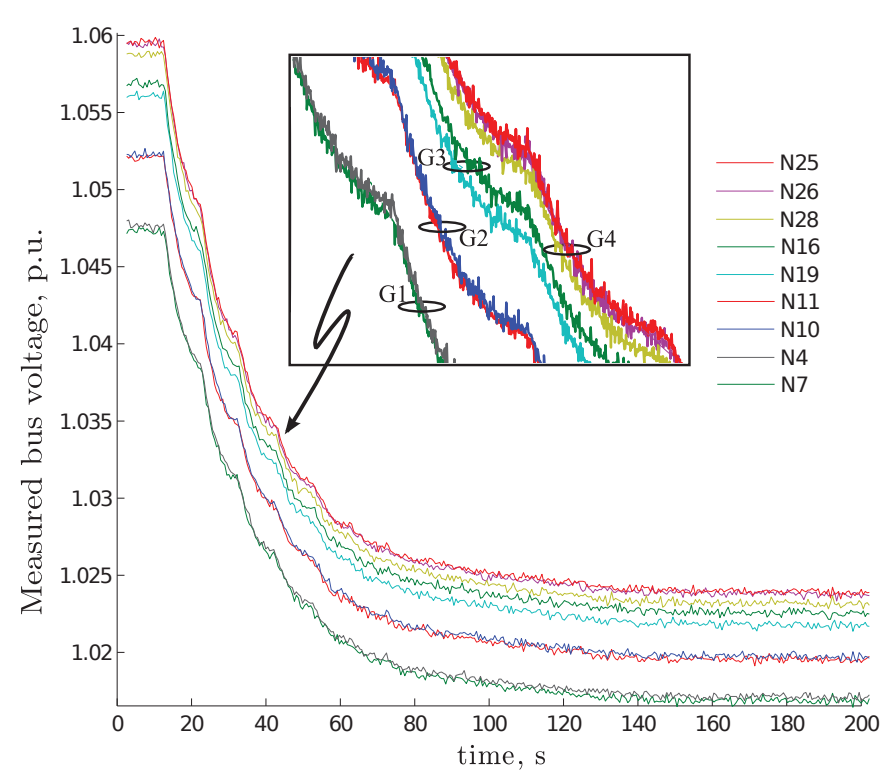

Figure 2. Evolution of bus voltages due to control actions

ten seconds the controller changes the power output of DG units to maintain the monitored bus voltages within limits, for details see [1].

Fig. 2 presents the smooth correction of an overvoltage condition using the centralized control scheme. The legends are placed in the same descending order as the plots.

Apart from the successful correction of the controller at $t=150 \mathrm{~s}$, it is important to observe that some bus voltages have similar evolution in time, identified by groups $G 1$ to $G 4$. The similarity of these bus voltages is directly related to bus location, load types and proximity to DG units.

Although bus voltages in the same group remain close one to another, it is more important to note that bus voltage changes belonging to the same group are remarkably similar. This observation is the basis of the proposed algorithm.

\section{Dissimilarity OF BUSES}

The estimation of a lost measurement requires the identification of similar buses that will be used to approximate bus voltage changes with respect to the last measured value. In this paper, similar buses refers to buses whose voltage evolution is similar, as shown in Fig. 2.

\section{A. Sensitivity Matrix}

The identification of groups of similar buses in a distribution network is obtained from the information provided by the sensitivity matrix $\mathbf{S}$. The latter contains the sensitivity of bus voltages with respect to power injections of DG units:

$$
\mathbf{S}=\left(\begin{array}{cccccc}
\frac{\partial V_{1}}{\partial P_{1}} & \cdots & \frac{\partial V_{1}}{\partial P_{g}} & \frac{\partial V_{1}}{\partial Q_{1}} & \cdots & \frac{\partial V_{1}}{\partial Q_{g}} \\
\vdots & \ddots & \vdots & \vdots & \ddots & \vdots \\
\frac{\partial V_{m}}{\partial P_{1}} & \cdots & \frac{\partial V_{m}}{\partial P_{g}} & \frac{\partial V_{m}}{\partial Q_{1}} & \cdots & \frac{\partial V_{m}}{\partial Q_{g}}
\end{array}\right)
$$

A large value in $\mathbf{S}$ matrix reflects the high impact of a power injection over a bus voltage. This sensitivity matrix is obtained from the inverse of the Jacobian matrix extracted from an offline power flow calculation [4]. The matrix will be seldom updated depending on the network operation, e.g. heavy or light loading conditions, or topology changes.

The $\mathbf{S}$ matrix may include other columns to provide more characteristics about the bus voltage evolutions. For instance, the sensitivity of bus voltages due to a transformer tap change can be added in a new column. This information can be easily extracted from the solution of two power flow runs with a single tap position difference.

The sensitivity matrix can be seen as a $m \times n$ data matrix of $m$ objects (bus voltages) and $n$ characteristics (sensitivity to power injections and tap changes). This matrix is used to find clusters of objects with similar characteristics. The groups can be identified based on dissimilarity measures, as will be explained in this Section.

\section{B. Dissimilarity Measures}

A dissimilarity measure quantifies the degree of distance between objects in $\mathbf{S}$. The distances are evaluated according to the object characteristics [5].

The classical distance measure is the Euclidean distance. Consider two objects from $\mathbf{S}$ matrix, $\alpha$ and $\beta$, with $n$ characteristics each. The Euclidean distance of these individuals is computed from the Pythagorean formula:

$$
d_{e}(\alpha, \beta)=\sqrt{\sum_{j=1}^{n}(\alpha(j)-\beta(j))^{2}}
$$

In case that $\alpha$ and $\beta$ had more than three characteristics, the distance could not be represented graphically in the $n$ dimensional space but it could still be computed.

There are other dissimilarity measures that may not represent a metric distance, but are useful to obtain the degree of dissimilarity between objects.

- Squared Euclidean Distance

The Squared Euclidean Distance (SED) is a dissimilarity measure that place greater weight on objects that are further apart. The SED for $\alpha$ and $\beta$ with $n$ characteristics is:

$$
d_{s}(\alpha, \beta)=\sum_{j=1}^{n}(\alpha(j)-\beta(j))^{2}
$$

\section{- Weighted Euclidean Distance}

The Weighted Euclidean Distance (WED) is of interest when $\mathbf{S}$ matrix contains characteristics that barely differ among objects while other attributes vary greatly from one individual to another.

To avoid dissimilarity results dominated by characteristics with large variations, a normalization with respect to the standard deviation of each characteristic is included [5]. The WED of objects $\alpha$ and $\beta$ in $\mathbf{S}$ is:

$$
d_{\omega}(\alpha, \beta)=\sqrt{\sum_{j=1}^{n}\left(\frac{\alpha(j)-\beta(j)}{\sigma_{j}}\right)^{2}}
$$


where $\sigma_{j}$ is the standard deviation of the $j t h$ characteristic in matrix $\mathbf{S}$ :

$$
\sigma_{j}=\sqrt{\frac{1}{m-1} \sum_{i=1}^{m}\left(\mathbf{S}(i, j)-\mu_{j}\right)^{2}}
$$

and $\mu_{j}$ is the mean value of the $j$ th characteristic:

$$
\mu_{j}=\frac{1}{m} \sum_{i=1}^{m} \mathbf{S}(i, j)
$$

Note that characteristics with small variations will be fairly considered in WED. In this paper, the Squared Weighted Euclidean Distance (SWED) is also evaluated:

$$
d_{s \omega}(\alpha, \beta)=\sum_{j=1}^{n}\left(\frac{\alpha(j)-\beta(j)}{\sigma_{j}}\right)^{2}
$$

In principle, SWED combines advantages of SED and WED.

The aforementioned distances will be used to calculate dissimilarities between bus voltages using the sensitivity matrix. Small distances means similar buses and this information will be used to obtain clusters of buses.

\section{Clustering and Selection of similar buses}

Bus clustering consists of grouping similar buses based on dissimilarity measures. These clusters will be used to approximate lost measurements using bus voltage measurements that belong to the same cluster. To do so, hierarchical clustering was chosen for finding groups of similar buses.

Hierarchical clustering uses the dissimilarity between objects to build hierarchy of clusters. The method is summarized as follows:

- Step 1: Examine all interpoint dissimilarities, and form a cluster from the two closest points.

- Step 2: Calculate the distance between the new cluster and any other point based on a linkage criterion.

- Step 3: Return to Step 1, treating clusters as well as remaining objects, until all objects are in one cluster.

In steps 1 and 2, "points" refers either to objects or clusters.

The distance between a new cluster and any point is calculated using the Lance-William's dissimilarity update formula [6]. This formula varies according to the linkage criteria. In this paper, the average link criterion was selected.

If points $\alpha$ and $\beta$ are agglomerated into cluster $\alpha \cup \beta$, the dissimilarity $d$ between the new cluster and any point $\gamma$ is:

$$
d(\alpha \cup \beta, \gamma)=\frac{|\alpha|}{|\alpha|+|\beta|} d(\alpha, \gamma)+\frac{|\beta|}{|\alpha|+|\beta|} d(\beta, \gamma)
$$

where $|\alpha|$ and $|\beta|$ are the number of objects in points $\alpha$ and $\beta$, while $d$ represents any of the dissimilarity measures mentioned above. Note that other update formulas allows other linkage methods to be implemented, for details see [6].

The resulting hierarchical clusters are generally represented in dendrograms [7]. These are tree diagrams used to illustrate the clusters and their hierarchy. These dendrograms consist

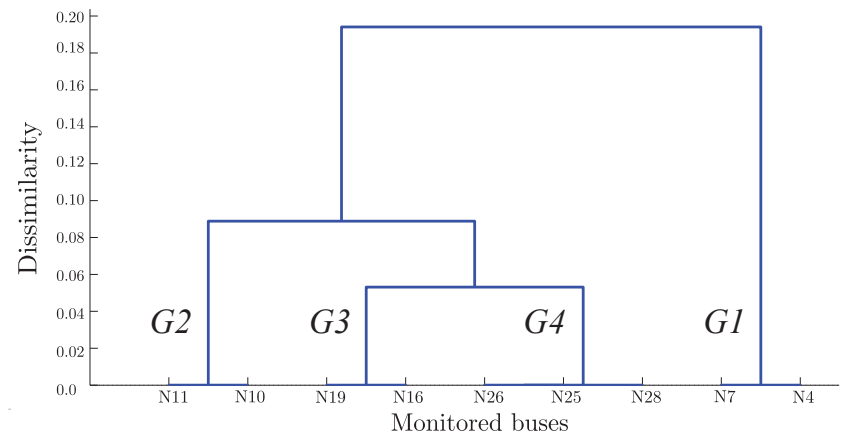

Figure 3. Dendrogram with SED

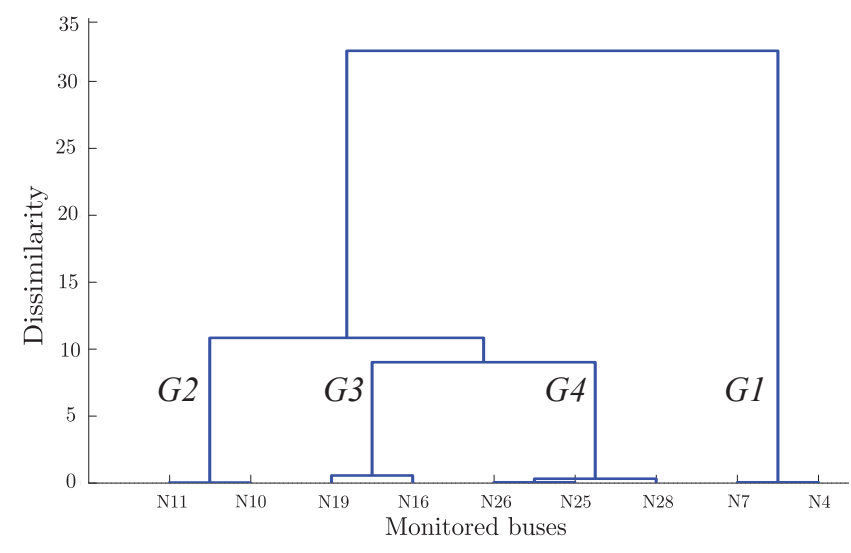

Figure 4. Dendrogram with SWED

of branches that connect objects in a hierarchical tree. The height of each branch represents the distance between the points connected. Hence, the highest branch corresponds to the largest dissimilarity between points.

Figs. 3 and 4 present the dendrograms of the monitored DN buses presented in Fig. 1, using the average link criterion with the SED and SWED dissimilarity measures.

From both dendrograms it is concluded that $N_{4}$ and $N_{7}$ are similar buses grouped in $G 1, N_{10}$ and $N_{11}$ form cluster $G 2$, buses $N_{16}$ and $N_{19}$ create cluster $G 3$ while $G 4$ is made of $N_{25}, N_{26}$ and $N_{28}$. Note that these clusters are in agreement with the groups identified in Fig. 2. Therefore, the clustering method based on the sensitivity matrix proves to be effective to identify bus voltages with similar evolutions.

The dendrogram also provides levels of dissimilarities. For example, buses $N_{16}$ and $N_{19}$ are similar buses but they are also similar, in a lower degree, to buses $N_{25}, N_{26}$ and $N_{28}$. Considering this hierarchy, if the voltage measurement of bus $N_{16}$ was lost, the proposed algorithm would primarily use available information of $N_{19}, N_{25}, N_{26}$ or $N_{28}$ to estimate the lost measurement. If none of these measurements were available, measurements from $N_{10}$ and $N_{11}$ would be used instead, resulting in less accurate estimations of the voltage evolution of $N_{16}$. This ability to identify levels of dissimilarity is the main benefit of hierarchical clustering.

The proposed algorithm selects the two closest buses available in the same or the immediate upper level of the dendro- 


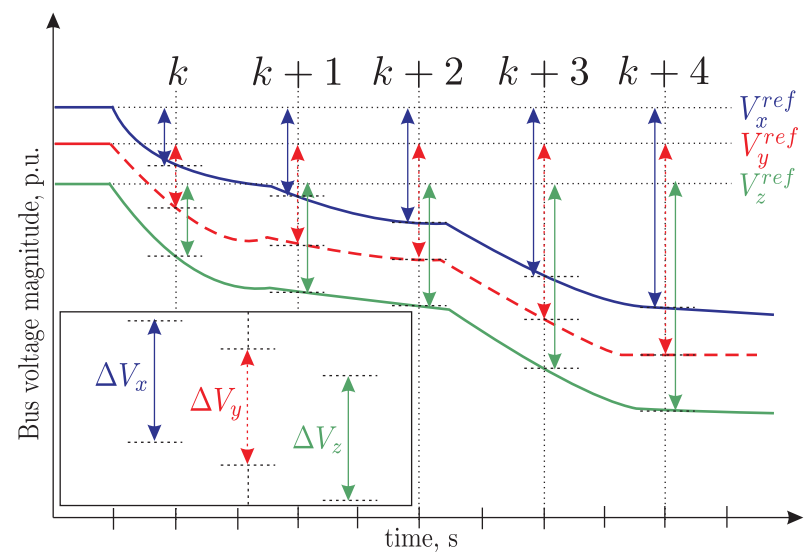

Figure 5. Reference voltage definition

gram. In case that voltage measurements of these buses are not available, the algorithm should select the next most similar buses (to the lost one) in the upper level of the dendrogram.

Consider the loss of voltage measurement $N_{10}$, according to the selection process, bus voltage measurements at bus $N_{11}$ and $N_{16}$ (closest object in the upper level) will be used to estimate the voltage evolution of $N_{10}$. To account for the levels of dissimilarity, the estimation should assign more weight to $N_{11}$ over $N_{16}$.

The dissimilarities between buses come in handy for calculating weight values. Suppose three monitored buses in a distribution network, $x, y$ and $z$, found to be similar buses. If bus voltage measurement $V_{y}$ gets lost, the weights assigned to $V_{x}$ and $V_{z}$ for estimating $V_{y}$ are:

$$
\begin{aligned}
& \omega_{y, x}=\frac{1}{d(y, x)} \\
& \omega_{y, z}=\frac{1}{d(y, z)}
\end{aligned}
$$

In this way, the most similar bus to $y$ will be assigned with a higher weight during the estimation process.

\section{Estimation OF LOST MEASUREMENTS}

Consider the evolution of the monitored buses $x, y$ and $z$ presented in Fig. 5. The three measurements $V_{x}, V_{y}$ and $V_{z}$ are sent to a controller until $V_{y}$ measurement is lost for instant $k$ and beyond, represented by the dashed line.

In order to estimate the evolution of $V_{y}$, the proposed method requires a set of reference voltages which are the last full set of voltage measurements (before the measurement loss occurrence), as presented in Fig. 5.

Since buses $x$ and $z$ were previously identified to have similar voltage evolutions to $y$, the change of $V_{y}$ should be similar to the change of $V_{x}$ and $V_{z}$, at any time instant. Based on this, the estimated evolution of $V_{y}$ at instant $k+i$ is:

$$
V_{y}(k+i)=V_{y}^{r e f}+\Delta V_{y}(k+i)
$$

where,

$$
\begin{gathered}
\Delta V_{y}(k+i)=\frac{\omega_{y, x} \Delta V_{x}(k+i)+\omega_{y, z} \Delta V_{z}(k+i)}{\omega_{y, x}+\omega_{y, z}} \\
\Delta V_{x}(k+i)=V_{x}(k+i)-V_{x}^{r e f} \\
\Delta V_{z}(k+i)=V_{z}(k+i)-V_{z}^{r e f}
\end{gathered}
$$

for $i=0,1, \ldots, l$, where $k+l$ refers to the last time instant before $V_{y}$ measurement is recovered. Note that $\Delta V_{y}$ is estimated as the weighted sum of $\Delta V_{x}$ and $\Delta V_{z}$.

According to (9) and (10), the most similar bus to $V_{y}$ will predominate in (12) over the least similar bus. For example, if the voltage measurement at $N_{16}$ is lost, the weights assigned to $N_{19}$ and $N_{25}$ in (12), using the SED, are:

$$
\Delta V_{N_{16}}=0.9998 \Delta V_{N_{19}}+0.0002 \Delta V_{N_{25}}
$$

for WED:

$$
\Delta V_{N_{16}}=0.7874 \Delta V_{N_{19}}+0.2036 \Delta V_{N_{25}}
$$

and for SWED:

$$
\Delta V_{N_{16}}=0.9394 \Delta V_{N_{19}}+0.0606 \Delta V_{N_{25}}
$$

Note that the use of squared distances results in higher weights assigned to the most similar bus. Hence, more accurate estimations should be obtained with SED and SWED.

From our experience, taking more than two buses to capture the evolution of a lost voltage measurement does not improve the approximations, as it will be shown in Section VI.

\section{Estimation Results}

This section presents the validation of the proposed method. For this purpose, simulated measurements of the 32-bus system under the influence of a centralized controller are used. The voltage measurements were simulated by adding white Gaussian noise restricted to $\pm 0.1 \%$.

The validation is based on comparisons of actual (simulated) bus voltage measurements and their estimated values. In all test cases, it is assumed that measurements are lost from the beginning of the simulation, so that the set of reference voltages is measured at $t=0 \mathrm{~s}$.

\section{A. Lost measurement estimation for Scenario 1}

This first scenario is the voltage correction presented in Fig. 2. As explained in Section II, the controller is able to smoothly bring all voltages within limits by changing the reactive power output of the DGs.

Figure 6 presents the estimation of $V_{7}$ using the information of the voltage evolution at buses $N_{4}$ and $N_{10}$. The estimations are very similar to the actual measurement, demonstrating the effectiveness of the algorithm with SED and SWED.

According to the dendrograms in Figs. 3 and 4, the evolution of $V_{7}$ is similar to $V_{4}$ and $V_{10}$. However, the proposed formulation gives higher weight to $V_{4}$ over $V_{10}$. 


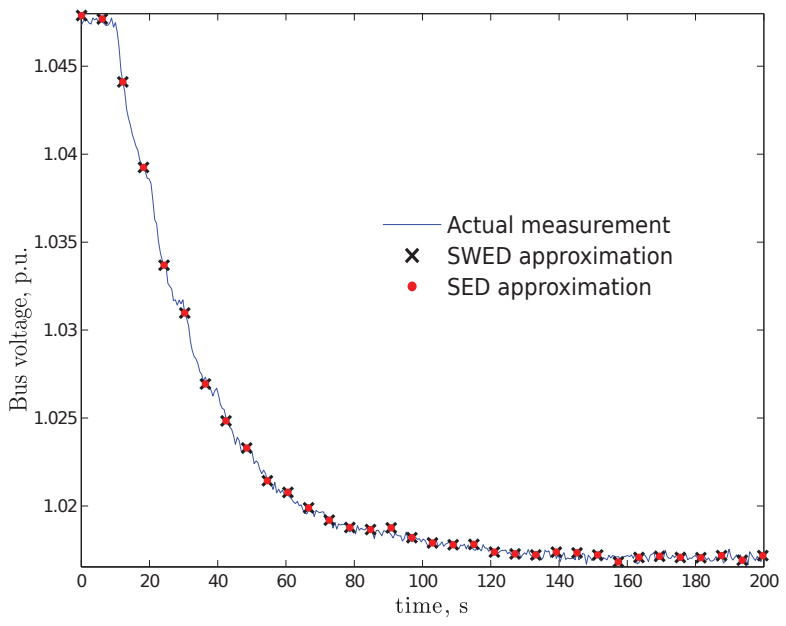

Figure 6. Estimation of $V_{7}$ using $V_{4}$ and $V_{10}$

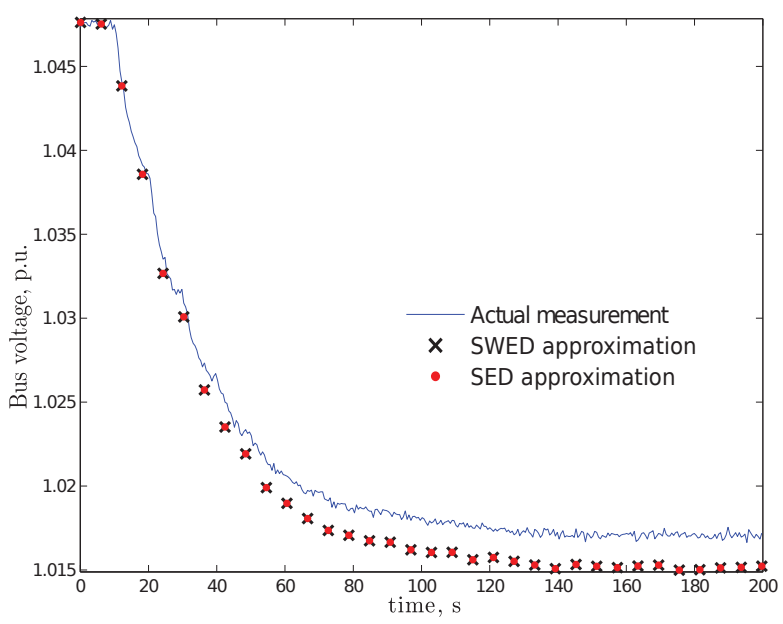

Figure 7. Estimation of $V_{7}$ using $V_{10}$ and $V_{11}$

In case that measurement $V_{4}$ is not available for estimating $V_{7}$, the algorithm will make use of the next most similar bus voltage measurements, $V_{10}$ and $V_{11}$.

Fig. 7 presents the estimation of $V_{7}$ when $V_{4}$ is also unavailable. Here, the use of less similar voltage measurements reduces the estimation accuracy of $V_{7}$. In addition, SED and SWED showed no significant estimation differences.

\section{B. Lost measurement estimation for Scenario 2}

Figure 8 presents a different voltage evolution of the monitored buses under the influence of the centralized controller. In this scenario, the controller brings all voltages close to 1.01 p.u. More details are presented in Case 2 of [3].

Fig. 9 and Fig. 10 show the estimation results of $V_{4}$ and $V_{26}$ respectively. In both cases, the estimations are remarkably similar to the actual measurement using the SED and SWED.

Figure 11 presents the estimation of $V_{16}$ using $V_{19}$ and $V_{25}$. Here, SED provides better results than SWED. This is explained because SED practically ignores the evolution of $V_{25}$, reflected in its lower weight assignation as presented in

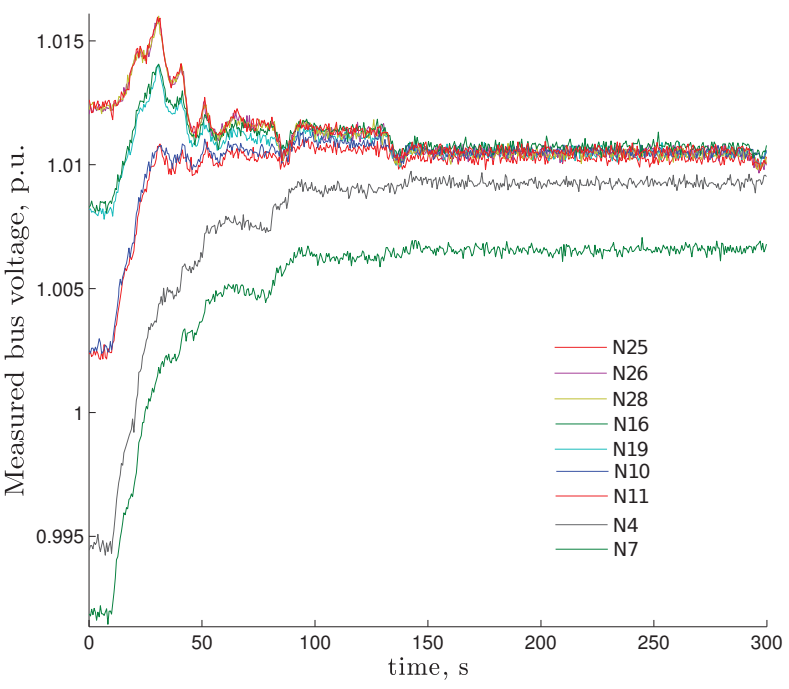

Figure 8. Evolution of bus voltages in Scenario 2

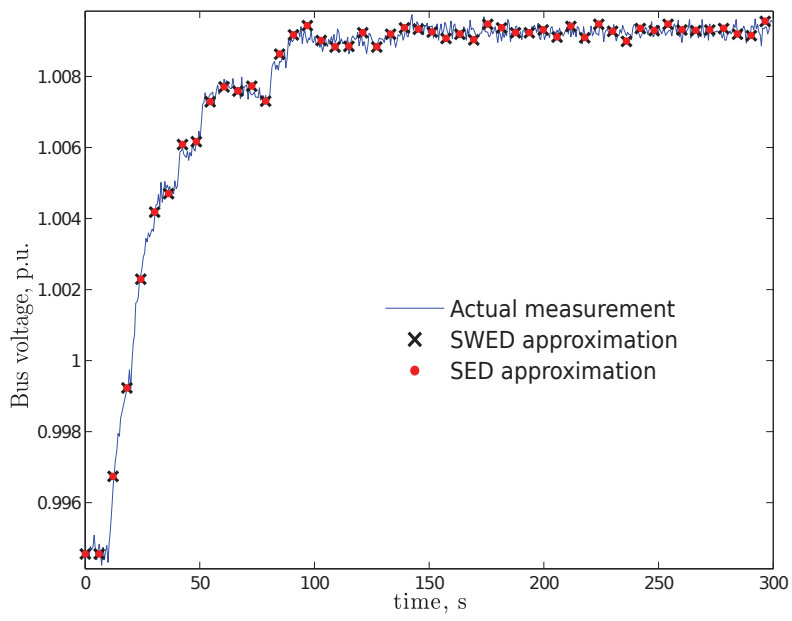

Figure 9. Estimation of $V_{4}$ using $V_{7}$ and $V_{10}$

Section V. In this particular case, the inclusion of $V_{25}$ slightly deteriorates the estimation of $V_{16}$.

\section{Effect of considering more than two measurements}

The last test is presented to analyze the impact of using more than two measurements to estimate a lost voltage measurement in Scenario 2. In this test, $V_{7}$ is estimated with two, five, and eight meters, following the dissimilarity levels of the dendrogram with SWED. Here, (12) was extended to consider five and eight meters.

Estimation results of $V_{7}$ are presented in Figure 12. It is observed that using more than two meters has little impact on the estimation results. This occurs because SWED highly discriminates the least similar buses in (12). Note that same results would be obtained with SED.

In case that WED is used, the estimation becomes less accurate as the weights are more distributed among meters, including the least similar ones. Figure 13 presents the estimation of $V_{7}$ in the case that WED is used. 


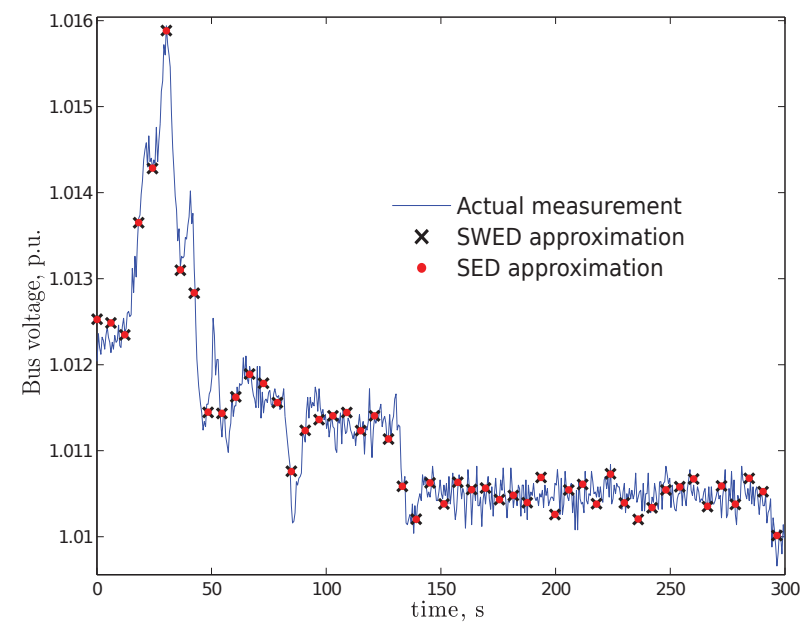

Figure 10. Estimation of $V_{26}$ using $V_{25}$ and $V_{28}$

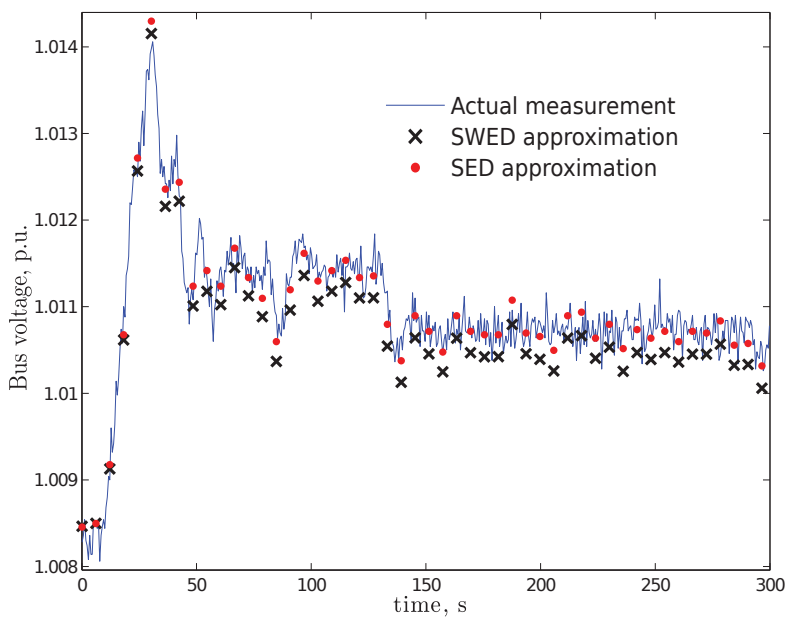

Figure 11. Estimation of $V_{16}$ using $V_{19}$ and $V_{25}$

Note that the accuracy is deteriorated for using measurements that are not similar to the lost one. From these tests it is concluded that using two meters is enough to capture the evolution of the lost measurement without compromising the accuracy of estimations.

\section{CONCLUSION}

This paper presents a simple algorithm that can be used for estimating voltage measurements that do not arrive to centralized control schemes. The algorithm principle is based on the observation that identifiable distribution bus voltages behave similarly under the influence of a centralized controller.

The paper showed that a sensitivity matrix can be used to identify clusters of buses with similar voltage evolutions. In addition, it was demonstrated that information from other measurements can be used to estimate lost measurements in balanced distribution networks.

This algorithm, to be coupled to a centralized controller, will automatically select the bus voltage measurements that best approximate the lost measurement. Future work will explore

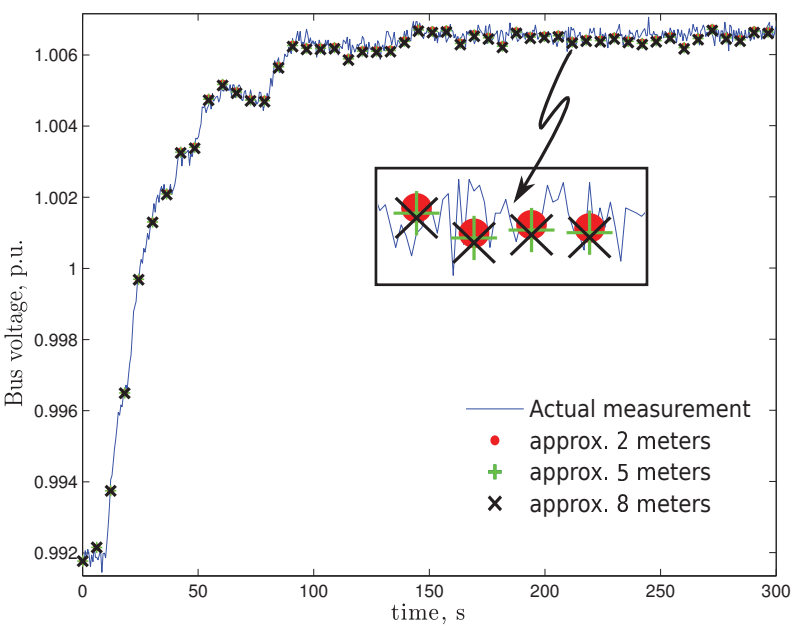

Figure 12. Estimation of $V_{7}$ using SWED with two and more meters

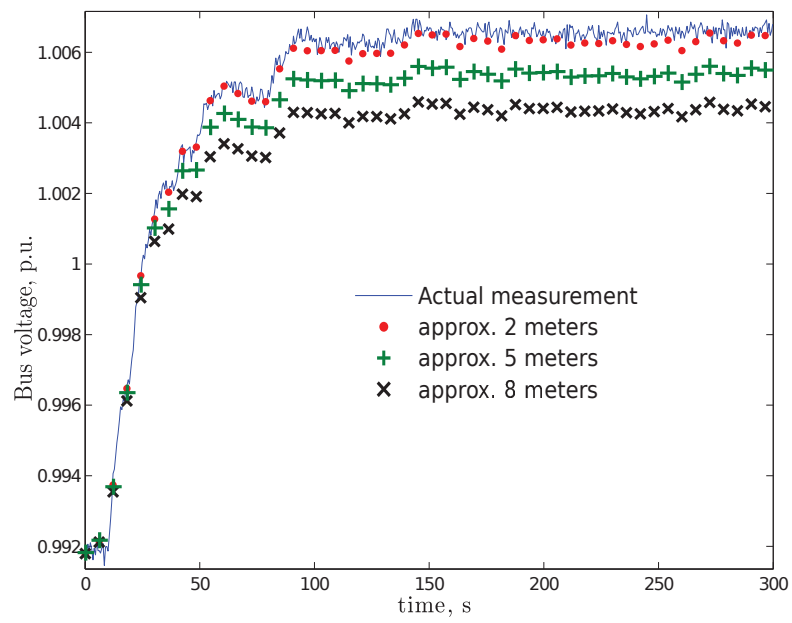

Figure 13. Estimation of $V_{7}$ using WED with two and more meters

the loss of multiple measurements and their effect on the controller performance.

\section{REFERENCES}

[1] G. Valverde and T. Van Cutsem, "Model predictive control of voltages in active distribution networks," IEEE Trans. Smart Grid, vol. 4, no. 4, pp. 2152-2161, Dec. 2013.

[2] S. Deshmukh, B. Natarajan, and A. Pahwa, "State estimation and voltagevar control in distribution network with intermittent measurements," IEEE Trans. on Smart Grid, vol. 5, no. 1, pp. 200-209, Jan. 2014.

[3] T. Van Cutsem and G. Valverde, "Coordinated voltage control of distribution networks hosting dispersed generation," in 22nd International Conference on Electricity Distribution, CIRED Stockholm, 2013.

[4] Q. Zhou and J. Bialek, "Generation curtailment to manage voltage constraints in distribution networks," IET, Generation, Transmission Distribution, vol. 1, no. 3, pp. 492-498, May 2007.

[5] P. Barrett, "Euclidean distance: raw, normalized, and double-scaled coefficients," Tech. Rep., 2005.

[6] F. Murtagh and P. Contreras, "Algorithms for hierarchical clustering: an overview," WIREs Data Mining and Knowledge Discovery, vol. 2, no. 1, pp. 86-97, Jan. 2012.

[7] R. Braddock, "Trees, dendrograms and sensitivity," in 1st biennial meeting of the International Environmental Modelling and Software Societey, Switzerland, 2002. 